\title{
基于红外成像的小型化稳定平台参数辨识
}

\author{
郭雪峰，林 宇 \\ (昆明物理研究所, 云南 昆明 650223)
}

\begin{abstract}
摘要: 针对红外成像的小型化稳定平台参数辨识的问题, 对系统中非线性因素进行尽可能抑制, 并在 高速环境下对系统进行辨识。在辨识过程中, 利用伪随机信号 (Pseudo random binary signal, PRBS) 作为调制的输入电压信号对系统激励, 相应的系统输出响应为速度信号, 并应用增广最小二乘方法辨 识线性离散模型参数, 最后通过参数化和非参数化两种方式, 对辨识模型的精确度进行了验证。结果 表明: 辨识得到的用来衡量实际系统输出随输入变化的传函关系的参数模型, 能够反映真实系统的动 态特性, 满足辨识精度要求。
\end{abstract}

关键词: 稳定平台; 参数辨识; 开环辨识; 增广最小二乘法

中图分类号：TP273 文献标识码：A＼cjkstart文章编号：1001-8891(2020)01-0030-10

\section{Parameter Identification for Miniature Stabilized Platforms Based on Infrared Imaging}

\author{
GUO Xuefeng, LIN Yu \\ (Kunming Institute of Physics, Kunming 650223, China)
}

\begin{abstract}
This study focuses on parameter identification for miniature stabilized platforms based on infrared imaging and offers a linearization technique to reduce the nonlinear effect on modeling identification. During the identification process, a pseudorandom binary sequence is used as the modulated input signal to drive the system, and the corresponding system output is a velocity signal. In addition, the augmented least square method is applied to identify the discrete modeling parameter. Finally, the precision of the modeling is tested in two ways: parameterization and non-parameterization. The results show that parameter modeling can adequately describe the dynamic character, similar to the real system.
\end{abstract}

Key words: stabilized platform, parameter identification, open-loop identification, augmented least square method

\section{0 引言}

基于红外探测成像的稳定平台是利用机电控制 达到隔离载体扰动, 从而保持红外探测器光轴/视轴在 惯性空间指向稳定的系统。为了保证对目标的成像质 量, 并且满足现今战场对武器体积小, 重量轻, 机动 特性好的需求 ${ }^{[1-2]}$, 在已经对小型化稳定平台结构优化 以及器件合理选型等的前提下, 为了能更大程度提升 后续动态性能和隔离性能, 就需要对系统的特性进行 研究, 再通过设计控制器进行改善。

小型化稳定平台是属于集平台载荷, 框架系统, 驱动与控制系统等于一体的复杂系统。如图 1 所示, 主要由 PWM (Pulse Width Modulation) 功率放大器、
直流电机、负载、传感器等共同组成, 整个控制系统 在输入信号作用下进行响应, 输出响应的类型和特征 能够反映系统特性。因此针对其复杂的系统组成, 只 能通过理论建模或实验建模两种方式对系统特性进 行描述 ${ }^{[3-4]}$ 。由于, 在平台的连接轴承、伺服电机电刷、 动摩擦件及导轨等部件中, 存在明显的非线性特征。 相比理论建模对于实际系统的刻画并不精确, 并且需 要繁重的推导过程等缺点, 实验建模并不完全依赖对 象先验知识的获取, 通过图表或参数方程都能反映系 统输入输出之间的关系。

在高频激励信号作用的动态辨识环境下, 除上述 非线性因素外, 包括残余噪声, 线绕力矩等在内的更 多误差源和非线性因素对系统的运动特性产生干扰, 
且不易用具体参数模型进行描述, 由此得到的伺服控 制系统的实际响应, 与根据各机电环节固有参数进行 理论推导的模型的响应相比差别很大, 用来辨识的输 入输出数据都是受非线性因素影响的非理想信息, 如 何利用受污染的数据辨识出无偏的参数, 这也是包括 稳定平台在内的机电系统辨识的共有难点。

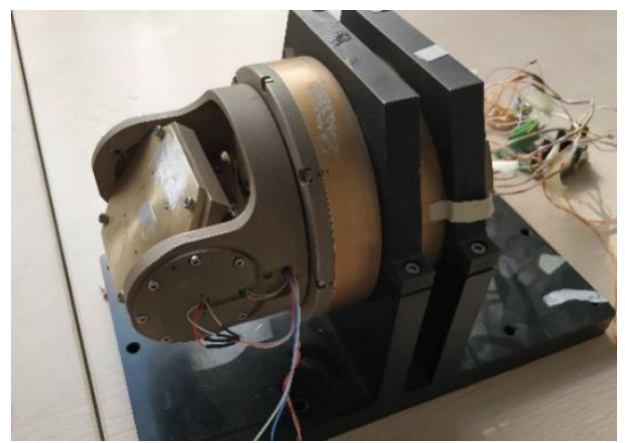

图 1 待辨识的稳定平台实物图

Fig.1 Actually stabilized platform to be identified

\section{1 小型化稳定平台辨识现状及分析}

针对小型稳定平台这类具有有限行程的机电系 统的辨识研究, 大多数文献都集中于对电机执行器件 的参数辨识, 如图 1 为待辨识的小型化稳定平台, 具 体问题表现在以下方面:

1）伺服系统工作时, 不可避免地需要克服因动 密封而引入的较大摩擦力矩和其他线绕力矩等非线 性因素, 基于 LTI（Liner time invariant）系统的时域 和频域辨识方法所用的速度或加速度数据都是受非 线性因素影响的非理想数据, 从而导致辨识结果产生 较大的误差。

2）现有的时域辨识算法通常采用充分激励后, 对采集得到的速度及加速度信息进行辨识, 小型稳定 平台因受行程范围的局限, 当在小幅值输入信号作用 下, 系统需要克服一定摩擦力, 而达不到能真实对应 输入信号作用下速度值, 当输入信号幅值过大时, 运 动范围超出有效行程而与框体撞击, 完全破坏固有的 运动状态, 采集到的信息完全不能表征原有系统, 而 使辨识失效, 同时, 一般的光电系统具有充分的运动 空间, 能够输入幅值足够大的激励信号, 相对能满足 更高的信噪比, 这也使得小型化稳定平台辨识易受量 化噪声的影响。

其次, 为了使辨识出的模型不仅具有与原系统近 似的动态特性, 还要保持一致的稳态值, 所以系统在 辨识过程中要有足够的空间位移渡过暂态过程, 进入 稳态过程, 但运动范围的限制会使系负载最终未能达 到稳态的速度终值, 这样辨识出的参数模型与真实系 统相比会有稳态误差。
3）应用优化算法在对非线性参数模型进行搜索 时, 不仅要对模型参数进行辨识, 如果以 stribeck 摩 擦模型为例对系统运动时摩擦特性进行描述时, 还要 对摩擦模型中的库伦摩擦力, 静摩擦力, 曲线系数, 防死区推力等参数进行辨识 ${ }^{[5]}$, 尤其当系统运行在不 同方向时, 要辨识的参数数目更是加倍, 辨识效率随 其数目的增多而大大降低, 算法很难收玫到能够满足 所有情况的全局最优值而失效, 辨识的参数也就无法 真实反映实际系统的物理参数。有必要对如何解决上 述依次存在的辨识问题, 进行讨论。

按照现已探索出的摩擦力与速度的函数模型, 如 图 2 所示。

基本所有机械系统都不可避免会受到非线性的 影响, 属于非线性系统。小型稳定平台由于要满足动 态快, 高精度等控制性能, 需要在高速状态下工作, 但受限于轻量化的机械结构特性, 使得转动惯量远低 于其他大型稳定平台, 在整个运动范围内很容易受摩 擦, 低频干扰等非线性因素的影响, 很难近似为一个 线性化系统进行描述。以摩擦力为例, 它是对系统运 动特性影响较大且不能彻底消除的因素之一, 用现已 探索出的摩擦力与速度的关系模型来描述, 可以分为 以下 4 个阶段:

I. 在静摩擦和预滑位移阶段, 由于预滑位移的存 在而产生了弹性变形, 因而表现出弹簧特性。此阶段 的摩擦力不依赖于速度, 主要体现与速度无关的静摩 擦特性。

II. 在边界润滑阶段, 摩擦力主要是由固体间的剪 切作用引起的, 其与低速爬行现象存在着密切的关 系。

III. 在部分流体润滑阶段, 随着滑动速度的增加润 滑膜变得越来越厚, 使得固体间突起的接触数量减 少。因而摩擦力呈现负斜率特性。

IV. 在全流体润滑阶段, 粘滞摩擦逐渐起主导作 用, 固体接触影响较小, 摩擦力会随着速度的增加而 增大。

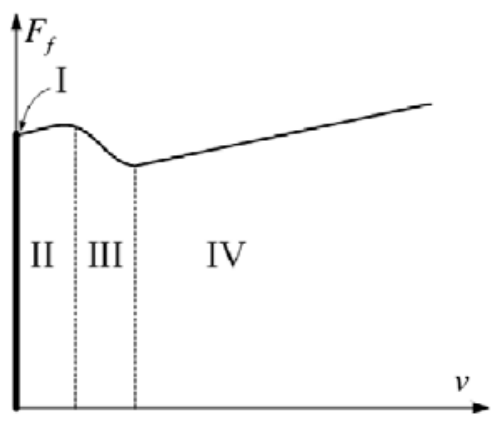

图 2 摩擦力与速度关系

Fig.2 Relationship between velocity and friction 
如果系统工作在低速区, 摩擦力受速度变化的影 响呈现明显的非线性特性, 工作在高速区时, 摩擦与 速度的关系可近似为比例关系, 如果在低速区对系统 辨识, 由于辨识出的参数化模型只能反映系统的线性 特征, 而非线性的干扰使得模型对原系统的刻画不精 确, 为了更大程度使系统受非线性影响减少, 要保证 在高速区对系统进行辨识。通常控制方式下, 待辨识 系统都会根据输入信号的幅值大小做出对应的速度 响应, 增大输入电压, 速度值也随之提高, 也就意味 着增大输入电压增益可以使系统工作在高速环境下。

这样, 通过一定线性化处理, 最大程度上避免非 线性因素影响, 并考虑将内部的各种非线性误差因素 看作系统中的统一的一个干扰源, 这部分对系统的影 响会叠加在系统理想输出上, 最终辨识得到的参数反 映输入信号与输出信号之间随时间变化的关系, 不考 虑内在变量的影响, 无需使用已有的摩擦模型进行描 述补偿 ${ }^{[5]}$, 也不用对多个随机的干扰进行单独建模分 析, 减少了不确定因素数量, 大大减小了辨识难度, 是在研究中可以实现的一种方法。

\section{2 伺服机构动力学建模}

动力学建模属于理论建模, 虽然这种方式对于实 际系统的刻画并不精确, 并且需要繁重的推导过程, 尤其是对于高阶多参数的系统更为不利, 但可以侧面 确定模型阶数, 是否含有积分环节等特征。待辨识对 象是由脉宽调制功率放大器、执行机构、负载、传感 器共同组成的复杂系统, 惯量盘作为唯一负载加在俯 仰框架上, 直流力矩电机和平台负载的等效电路图如 图 3 所示。

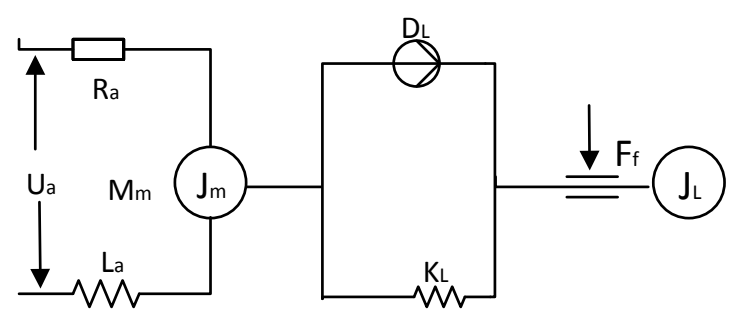

图 3 电机和负载等效电路图

Fig.3 Equivalent diagram of motor and load

由直流力矩电机电枢的电压平衡方程、反电势方 程和力矩电机电磁作用方程可得:

$$
\begin{aligned}
U_{\mathrm{a}}(t)= & R_{\mathrm{a}} I_{\mathrm{a}}(t)+L_{\mathrm{a}} \dot{I}_{\mathrm{a}}(t)+\varepsilon(t) \\
& \varepsilon(t)=K_{\mathrm{e}} \dot{\theta}_{\mathrm{m}}(t) \\
& T_{\mathrm{m}}=K_{\mathrm{m}} i_{\mathrm{a}}(t)
\end{aligned}
$$

式中: $U_{\mathrm{a}}$ 为电枢电压; $\varepsilon$ 为反电动势; $i_{\mathrm{a}}$ 为电机电枢电
流; $R_{\mathrm{a}}$ 为电枢电阻; $L_{\mathrm{a}}$ 为电枢电感; $K_{\mathrm{e}}$ 为反电势系数; $\theta_{\mathrm{m}}, \dot{\theta}_{\mathrm{m}}, \ddot{\theta}_{\mathrm{m}}$ 分别为电机轴的输出转角、角速度和角加速 度; $T_{\mathrm{m}}$ 为电机输出转矩; $K_{\mathrm{m}}$ 为电机力矩系数, $\mathrm{N} \cdot \mathrm{m} / \mathrm{A}$ 根据电机系统的功率平衡原理, 在数值上有关系式 $K_{\mathrm{e}}=K_{\mathrm{m}}$ 成立。

由电机动力学原理可得:

$$
\begin{gathered}
J_{\mathrm{m}} \ddot{\theta}_{\mathrm{m}}+D_{\mathrm{L}}\left(\dot{\theta}_{\mathrm{m}}(t)-\dot{\theta}_{\mathrm{L}}(t)\right)+D_{\mathrm{L}}\left(\theta_{\mathrm{m}}(t)-\theta_{\mathrm{L}}(t)\right)=T_{\mathrm{m}}(t) \quad(4) \\
J_{\mathrm{m}} \ddot{\theta}_{\mathrm{L}}+D_{\mathrm{L}}\left(\dot{\theta}_{\mathrm{m}}(t)-\dot{\theta}_{\mathrm{L}}(t)\right)+K_{\mathrm{L}}\left(\theta_{\mathrm{m}}(t)-\theta_{\mathrm{L}}(t)\right)+F_{\mathrm{f}}(t)=0
\end{gathered}
$$

式中: $J_{\mathrm{m}}$ 和 $J_{\mathrm{L}}$ 分别为电机和负载的转动惯量; $D_{\mathrm{L}}$ 和 $K_{\mathrm{L}}$ 分别为系统的阻尼系数和刚度系数; $F_{\mathrm{f}}$ 为传动链上 的摩擦力矩; $\theta_{\mathrm{L}}, \dot{\theta}_{\mathrm{L}}, \ddot{\theta}_{\mathrm{L}}$ 分别为负载轴的输出转角、角 速度和角加速度。

同时, 所用的 PWM 式功率放大器的带宽远大于 控制系统的工作带宽, 其用来表示输出整流电压与微 小控制输入电压之间的函数关系可以忽略延迟, 仅用 下式表达:

$$
\begin{gathered}
U_{\mathrm{a}}(t)=R_{\mathrm{a}} I_{\mathrm{a}}(t)+L_{\mathrm{a}} \dot{I}_{\mathrm{a}}(t)+\varepsilon(t) \\
G_{\mathrm{pwm}}(\mathrm{s})=k_{\mathrm{pwm}}
\end{gathered}
$$

式中: $k_{\mathrm{pwm}}$ 表示 PWM 功率放大器的对输入电压的放 大系数。摩擦是通过速度对电枢电流响应施加干扰 的。当系统在单向宏观滑动运动时 (即增大增益, 使 对象快速度过启动期的非线性摩擦力作用区），这时 摩擦工作特性可用下式简单的库伦摩擦及粘滞摩擦 描述 ${ }^{[6]}$, 使速度满足阈值条件, 将摩擦尽可能线性化 为常值耦合到模型参数中辨识:

$$
F_{\mathrm{f}}=\left\{\begin{array}{cc}
F_{\mathrm{C}}^{+} & \dot{\theta}_{0}(t)>\dot{\theta} \varepsilon \\
\min \left(F_{\mathrm{d}}, F_{\mathrm{s}}\right) & \left|\dot{\theta}_{0}(t)\right| \leq \dot{\theta} \varepsilon \\
F_{\mathrm{C}}^{-} & \dot{\theta}_{0}(t)<\dot{\theta} \varepsilon
\end{array}\right.
$$

式中: $F_{\mathrm{C}}{ }^{+}$表示正向运动的库仑摩擦力; $F_{\mathrm{C}}{ }^{-}$表示反向 运动的库仑摩擦力; $F_{\mathrm{s}}$ 表示静摩擦力; $\dot{\theta}_{\varepsilon}$ 为临界角速 度; $\dot{\theta}_{\varepsilon}(t)$ 为当前时刻的负载运动速度, 由于辨识的 目的不仅在于用更准确的参数模型去表达实际系统, 还需要用更精简 (低阶) 的结构来表示, 虽然存在电 机和负载之间非刚性因素以及摩擦力的作用, 但因为 不考虑系统内部状态, 经过线性化处理, 参数模型只 单纯表征输入输出的关系, 其中参数不是代表电机的 固有参数, 可以将它们对系统响应的影响折算到模型 的参数上来反映, 所以由微小控制电压 $u$ 到负载轴输 出角速度 $\dot{\theta}_{\mathrm{L}}$ 之间的传递函数模型用如下二阶模型表 
示:

$$
G_{\mathrm{p}}(s)=\frac{a s+b}{c s^{2}+d s+e}
$$

则只需对 $a, b, c, d, e$ 这 5 个参数进行辨识, 就可以直接用来表征电机携负载从输入电压信号到 输出速度响应的参数模型。

\section{3 开环辨识分析与实验}

开环条件下，系统会按照所施加电压信号随时间 变化的规律做出直观响应, 由于缺少反馈的引入, 稳 定平台的俯仰框架上的负载运动只能由输入信号一 个变量控制, 考虑到平台伺服机构存在的有限行程限 制, 故对光电稳定平台而言, 其辨识过程还需满足以 下约束条件:

$$
\begin{gathered}
\operatorname{abs}\left(\dot{\theta}_{0}(t)\right) \leq \dot{\theta}_{\max } \\
\theta_{\text {lower }} \leq \theta_{0}(t) \leq \theta_{\text {upper }}
\end{gathered}
$$

式中: $\dot{\theta}_{\text {max }}$ 表示系统允许最大速度; $\theta_{\text {lower }}$ 和 $\theta_{\mathrm{upper}}$ 表示 平台角位置的下界和上界, 分别为 $-85^{\circ}$ 和 $+85^{\circ}$ 。由 于轻量化需求, 平台系统不再配置专门的测速装置, 速度信息直接由位置信号差分估计得到, 模数转换器 的滤波作用能避免速度量化噪声对辨识结果的影响。

稳定平台伺服系统中，输入为微小控制电压 $u$, 输出为负载端的转速信号 $\dot{\theta}_{L}$, 由公式(9)可得这是一个 2 阶的连续系统模型, 而在参数辨识过程中, 计算机 是对系统输入输出采样后的离散信号进行处理, 则最 终辨识得到的也是离散化模型, 转换后待辨识的离散 化模型为:

$$
H(z)=\frac{b_{1} z+b_{2}}{a_{1} z^{2}+a_{2} z+a_{3}}
$$

其中的参数不再与前述分析的连续模型的参数 一一对应。

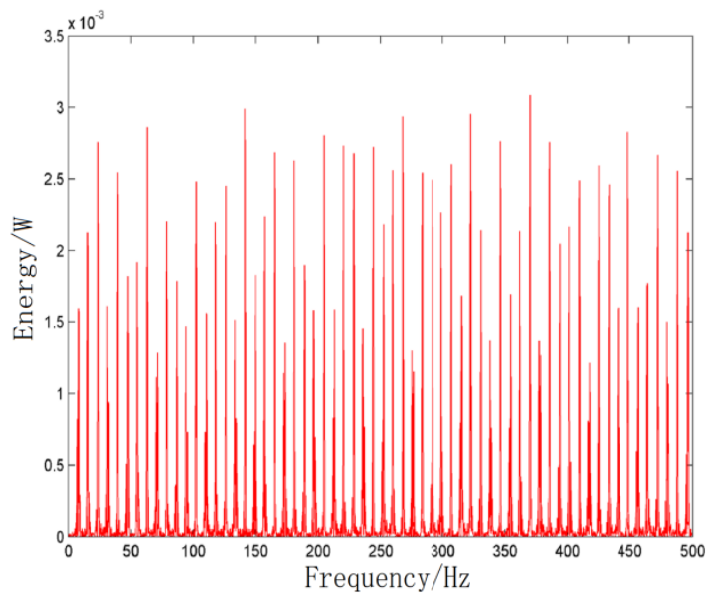

(a) $1000 \mathrm{~Hz}$ 的 1 分频的 PRBS 的 PSD 分析

(a) PSD analysis of PRBS in 1 frequency division of $1000 \mathrm{~Hz}$
用做激励的伪随机信号（PRBS）是由矩形脉宽 组成的随机序列, 单个的矩形脉宽的周期决定了整个 序列段最大的变化频率 $f_{\text {max }}$, 参考带宽的范围, 最终 辨识的模型在此范围内的动态特性要与实际系统近 似, 因此, 在此频率范围内, 激励信号的能量要较强, 以满足充分激励的条件，同时高频区能量要低，从而 对高频分量不激励。

PRBS 的能量分布与其变换频率相关, 通过功率 谱密度 (Power Spectral Density, PSD) 分析能看出变 换频率与能量分布的关系, 在采样率 $f_{\mathrm{w}}$ 分别为 1000 $\mathrm{Hz} 、 500 \mathrm{~Hz} 、 333 \mathrm{~Hz}$ 的情况下，采集 PRBS 的变换频 率分别为不同采样率 $f_{\mathrm{w}}$ 的 $1,1 / 2,1 / 3$ 倍（即分频, $1 / 2$ 倍代表 2 分频)时的 PRBS(位数为 8, 幅值为 0.1 ), 然后进行 PSD 分析, 结果如图 4 所示。

可以看出, 相对于不同采样率的相同分频数, 激 励信号的能量分布特性一致, 不分频时, 能量在 $f_{\mathrm{w}}$ 范 围内均匀分布, 2 分频时大概在 $f_{\mathrm{w}}$ 的 $0 \sim 0.35$ 的范围 内能量比较强, 剩余区域能量较弱, 3 分频时会在 $f_{\mathrm{w}} / 3$ 处有断档。在采样率为参考的基础上，对伪随机信号 进行 2 分频处理。可以使高频能量衰减，对带宽范围 所属的低频区激励较多。

对于 PRBS 的位数和幅值的选择, 要结合对象的 运动范围考虑。如果 PRBS 的正负脉宽数量不均匀, 惯量盘就会随时间增长向某一方向靠近而撞击到轴 挡, 而不是在工作点附近来回运动, 此实验中待辨识 的小型化稳定平台主要是电机驱动负载在俯仰方向 内运动，上下有限位的设置，分别在正负 $85^{\circ}$ 角的位 置处, 以防止连续旋转使线绕断裂，所以对象的运动 范围也就被限制在其中, 图 5 即为负载分别在俯仰框 内 $0^{\circ}, 45^{\circ}, 85^{\circ}$ 的示意图。

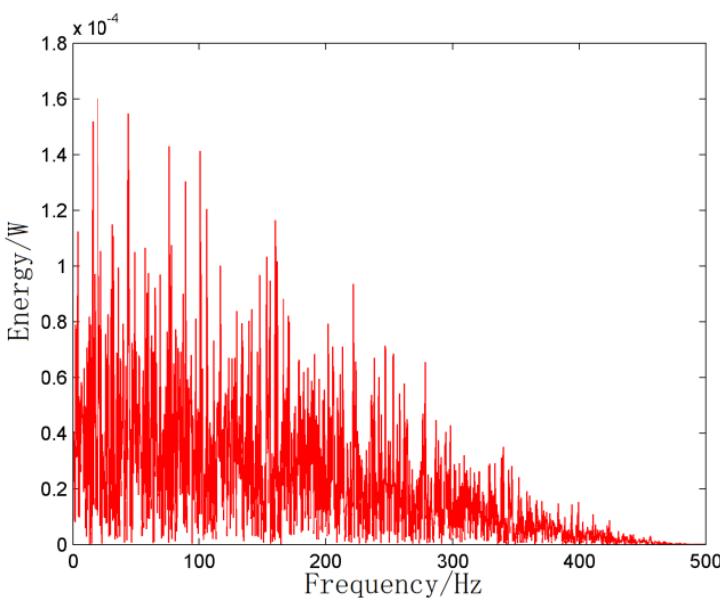

(b) $1000 \mathrm{~Hz}$ 的 2 分频的 PRBS 的 PSD 分析

(b) PSD analysis of PRBS in 2 frequency division of $1000 \mathrm{~Hz}$ 


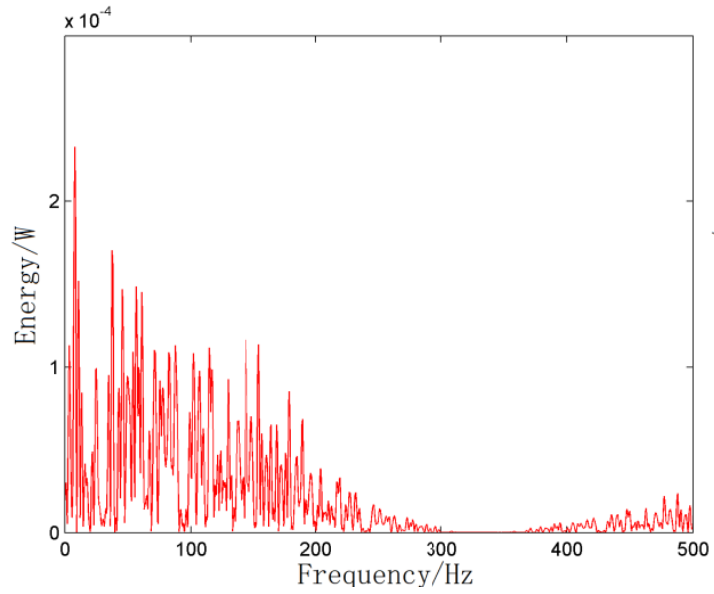

(c) $1000 \mathrm{~Hz}$ 的 3 分频的 PRBS 的 PSD 分析

(c) PSD analysis of PRBS in 3 frequency division of $1000 \mathrm{~Hz}$

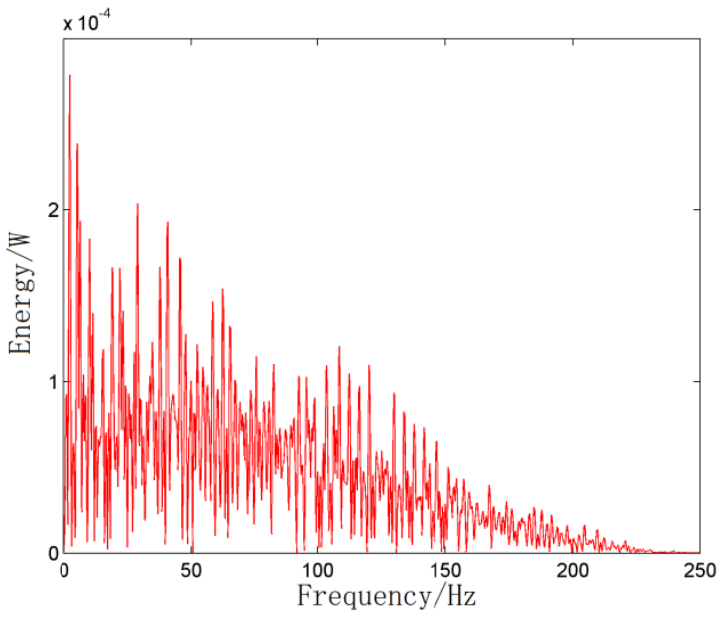

(e) $500 \mathrm{~Hz}$ 的 2 分频的 PRBS 的 PSD 分析

(e) PSD analysis of PRBS in 2 frequency division of $500 \mathrm{~Hz}$

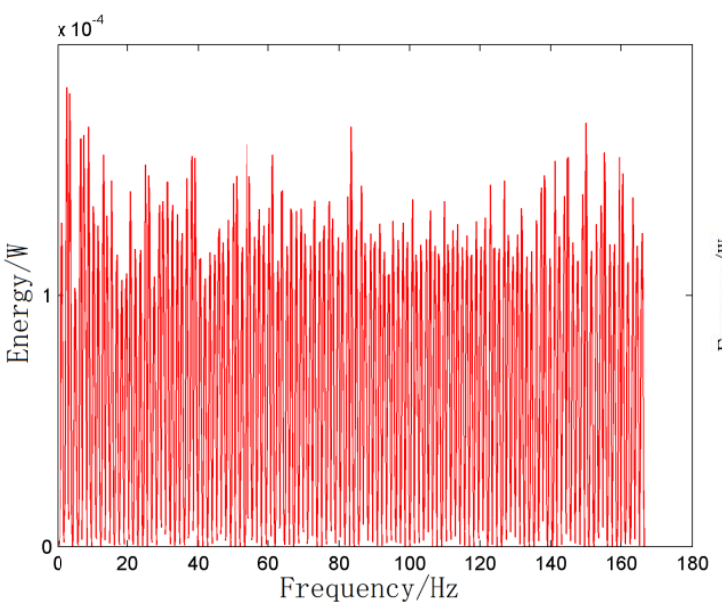

(g) $333 \mathrm{~Hz}$ 的 1 分频的 PRBS 的 PSD 分析

(g) PSD analysis of PRBS in 1 frequency division of $333 \mathrm{~Hz}$

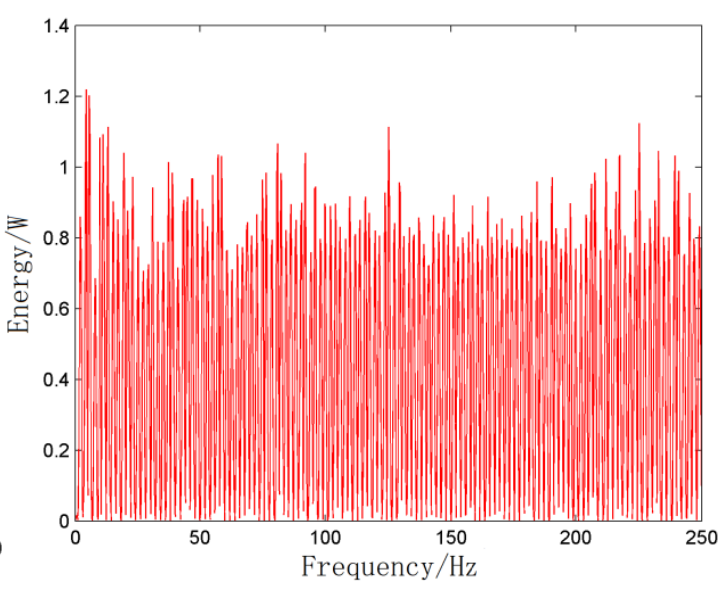

(d) $500 \mathrm{~Hz}$ 的 1 分频的 PRBS 的 PSD 分析

(d) PSD analysis of PRBS in 1 frequency division of $500 \mathrm{~Hz}$

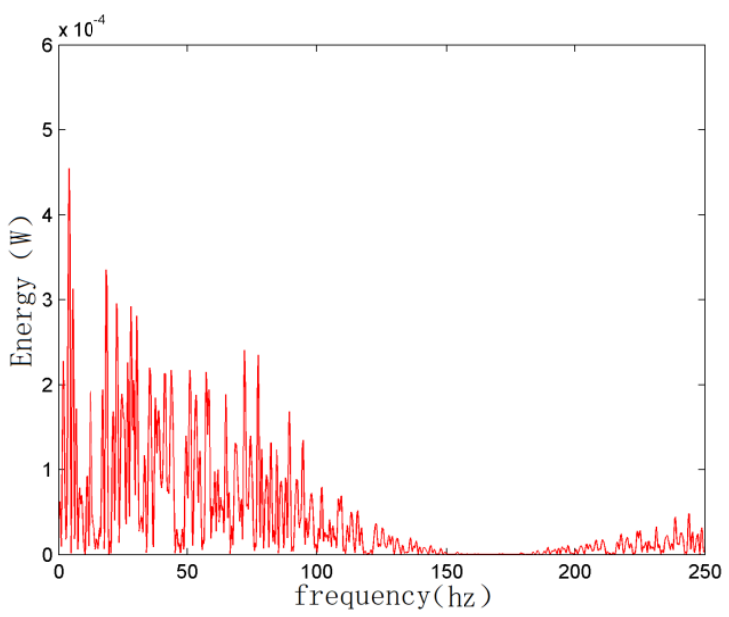

(f) $500 \mathrm{~Hz}$ 的 3 分频的 PRBS 的 PSD 分析

(f) PSD analysis of PRBS in 3 frequency division of $500 \mathrm{~Hz}$

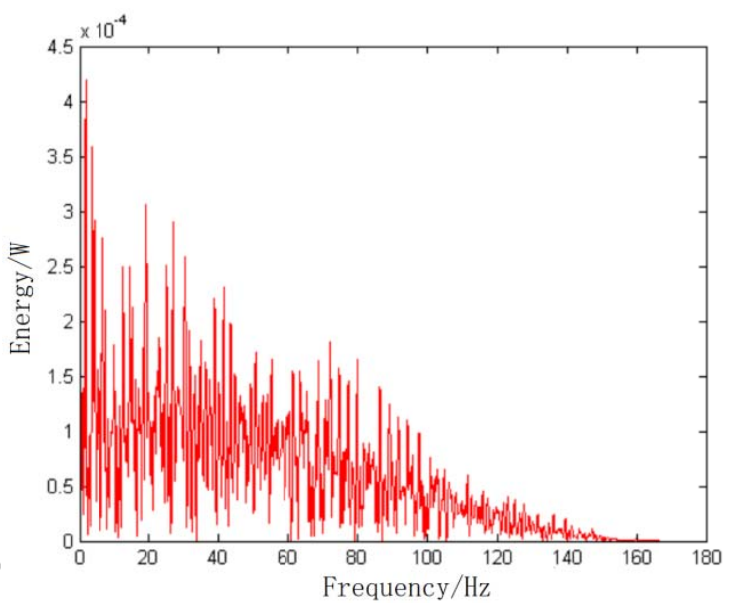

(h) $333 \mathrm{~Hz}$ 的 2 分频的 PRBS 的 PSD 分析

(h) PSD analysis of PRBS in 2 frequency division of $333 \mathrm{~Hz}$ 


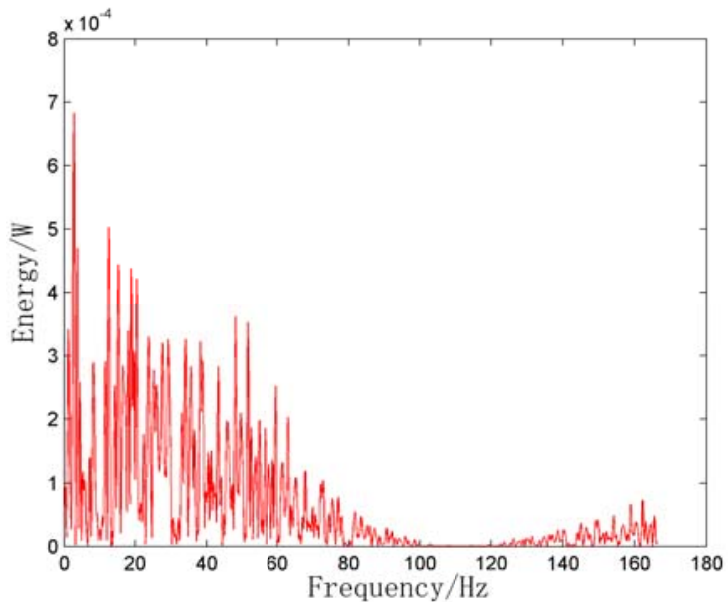

(i) $333 \mathrm{~Hz}$ 的 3 分频的 PRBS 的 PSD 分析

(i) PSD analysis of PRBS in 3 frequency division of $333 \mathrm{~Hz}$

图 4 不同变换频率 PRBS 的 PSD 分析

Fig.4 PSD analysis of PRBS used in different change frequency condition

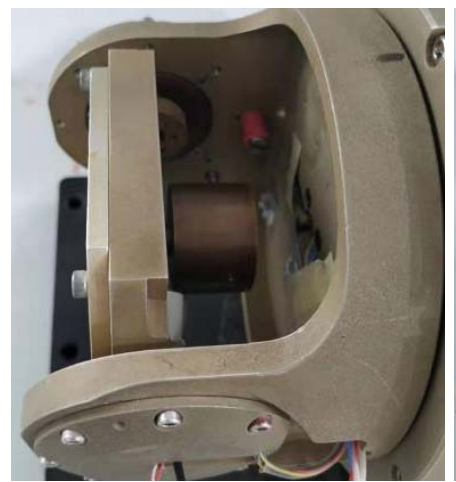

(a) 俯仰 $0^{\circ}$

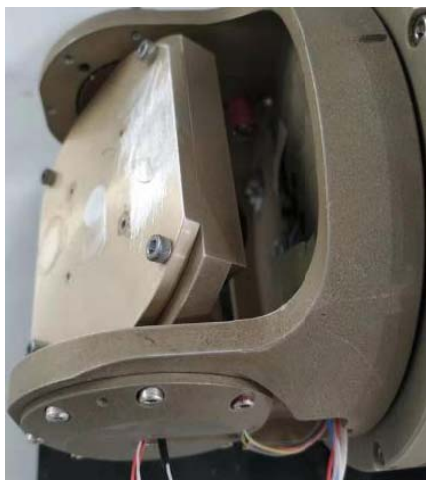

(b) 俯仰 $45^{\circ}$

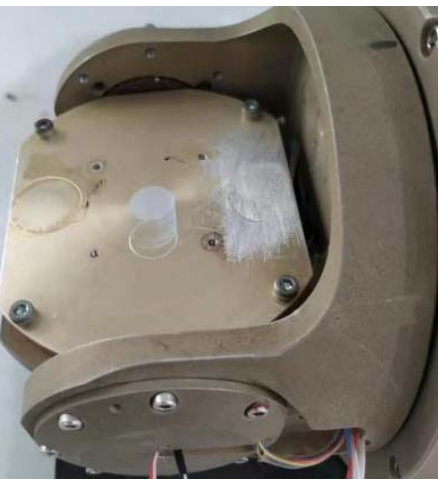

(c) 俯仰 $85^{\circ}$

(a) pitch 0 degree

(b) pitch 0 degree

(c) pitch 0 degree

图 5 负载分别在俯仰框内位置 $0^{\circ}, 45^{\circ}, 85^{\circ}$ 的示意图

Fig.5 Actual load position in 0degree, 45degree, 85degree respectively

通过测试, 由 7 位和 8 位 PRBS 产生的一个周期 内的序列里的正负脉宽的数量比较对称。

PRBS 信号的序列长度 $L$ 满足下式:

$$
L=2^{n-1}
$$

式中: $n$ 为寄存器数。

除此之外辨识前要进行数据预处理, 对 $\mathrm{I} / \mathrm{O}$ 数据 进行去除对应工作点的直流分量的工作。主要分两 步:

1）计算 $I / O$ 数据的平均值。

2 ) 从 $\mathrm{I} / \mathrm{O}$ 数据中减去平均值, 得到新的 $\mathrm{I} / \mathrm{O}$ 数据。

\section{4 实验验证}

搭建数字控制系统进行系统辨识的实验和验证 工作。数字系统主要由直流电机、旋转变压器、惯量 盘、功率放大器和 Dspace 数字控制平台组成。图 6
为以俯仰方向为例的伺服系统控制框图, 其中对稳定 平台进行伺服控制的功率放大器的电压调制输入信 号通过 Dspace 设备提供, 旋转变压器用来测量俯仰 轴的转角信息, 信号采集解算卡对旋变模拟信号进行 采集, 按照 16 位的分辨率进行解算, 并按照数据组 合据协议进行处理后, 由 Dspace 接收并传输给上位 机。

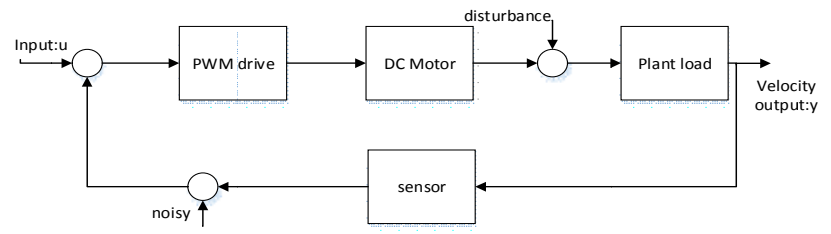

图 6 稳定平台的系统控制框图

Fig.6 Single-axis system block diagram of stabilized platform 4.1 交叉实验和白噪化验证 
设计交叉实验, 用来确定 PRBS 经过 PSD 分析后 的能量分布对高低频率区间的激励如何分配时，可以 使辨识出的参数模型的动态特性更贴近真实系统, 然 后选取通过白噪化验证的模型进行非参数化验证。其 中白噪化, 是利用两个信号之间的误差的自相关性来 描述他们响应的相似程度, 进而反映实际对象与模型 的差异大小 ${ }^{[7]}$ 。如果在时间零点两者的相关值为理想 的 1 , 而在不同时间间隔 $\tau$ 下比对的相关值减小, 则表 明两者相关性很高, 系统响应近似相等。

通过下述的验证准则来判别是否模型有效:

$$
\left|R_{N}(i)\right| \leq \frac{2.17}{\sqrt{N}} \quad i \geq 1
$$

式中: $N=200$ 为采样数, 交叉实验结果和分析如表 1 所示。

可以看出: 激励信号对系统带宽范围内的频率成 分起到激励作用, 但是要对带宽以外的部分 $(\omega \sim 3 \omega$ 范围) 起到部分激励时, 预测模型能够通过验证。太 高采样率会引入高频噪声, 并且使辨识过程中观测向 量 $\psi^{\mathrm{T}} \psi$ 之间的数值条件变差, 如果用降低采样率而不
经分频处理的 PRBS 信号激励, 在整个 $0 \sim 0.5 f$ 区域 内的能量均匀分布, 对此范围内频率成分会均匀激 励, 高频区也会得到更多激励, 对辨识结果有影响, 最终未能通过验证根据交叉实验结果, 通过白噪化验 证的离散化参数模型为:

$$
\frac{y_{u}(z)}{u(z)}=\frac{21.537 z+107.1297}{z^{2}-1.0688 z+0.0982}
$$

成形滤波器的模型如下:

$$
\frac{n(z)}{u(z)}=\frac{z^{2}-0.0806 z-0.0054}{z^{2}-1.0688 z+0.0982}
$$

式中: $y_{u}(z)$ 为系统的系统理想输出; $u(z)$ 为系统理想 输入; 实际输出 $y(z)=y_{u}(z)+n(z) ; n(z)$ 是通过 $v(z)$ 经 成形滤波器形成的干扰; $v(z)$ 是均值为 0 的白噪声,

在辨识中是用预测误差 $\dot{v}(\mathrm{z})=e(\mathrm{z})$ 来替代。

图 7 展示了辨识过程中, 在采样率为 $3 \mathrm{~ms}$ 时, 经 2 分频处理后的 PRBS 激励下的速度响应曲线。图 8 展示了实际系统与通过白噪化验证的参数模型的速 度输出响应在 200 个采样点下的误差曲线。

\begin{tabular}{|c|c|c|c|c|c|}
\hline Sampling rate/ms & $\begin{array}{l}\text { Frequency division } \\
\text { number }\end{array}$ & $\begin{array}{l}\text { Order of } \\
\text { system }\end{array}$ & $\begin{array}{l}\text { Amplitude of the exciting } \\
\text { signal (PRBS) }\end{array}$ & $\begin{array}{l}\text { Whitening Test: } \\
\text { R0 is theoretical value R1 is } \\
\text { actual value } N=200\end{array}$ & $\begin{array}{l}\mathrm{R} 1<\mathrm{R} 0 \text { indicate } \\
\text { Whitening Test pass }\end{array}$ \\
\hline \multirow[t]{2}{*}{1} & 1 & 2 & 0.3 & $\mathrm{R} 0=0.1418$ & No \\
\hline & & & & $\mathrm{R} 1=0.4896$ & \\
\hline \multirow[t]{2}{*}{1} & 2 & 2 & 0.3 & $\mathrm{R} 0=0.1418$ & No \\
\hline & & & & $\mathrm{R} 1=0.5525$ & \\
\hline \multirow[t]{2}{*}{1} & 4 & 2 & 0.3 & $\mathrm{R} 0=0.1418$ & No \\
\hline & & & & $\mathrm{R} 1=0.8631$ & \\
\hline \multirow[t]{2}{*}{1} & 6 & 2 & 0.3 & $\mathrm{R} 0=0.1418$ & No \\
\hline & & & & $\mathrm{R} 1=0.1970$ & \\
\hline \multirow[t]{2}{*}{3} & 1 & 2 & 0.3 & $\mathrm{R} 0=0.1418$ & No \\
\hline & & & & $\mathrm{R} 1=0.6028$ & \\
\hline \multirow[t]{2}{*}{3} & 2 & 2 & 0.3 & $\mathrm{R} 0=0.1418$ & Yes \\
\hline & & & & $\mathrm{R} 1=0.1177$ & \\
\hline \multirow[t]{2}{*}{3} & 3 & 2 & 0.3 & $\mathrm{R} 0=0.1418$ & No \\
\hline & & & & $\mathrm{R} 1=0.5625$ & \\
\hline \multirow[t]{2}{*}{6} & 1 & 2 & 0.3 & $\mathrm{R} 0=0.1418$ & No \\
\hline & & & & $\mathrm{R} 1=0.4785$ & \\
\hline \multirow[t]{2}{*}{6} & 2 & 2 & 0.15 & $\mathrm{R} 0=0.1418$ & No \\
\hline & & & & $\mathrm{R} 1=0.5502$ & \\
\hline
\end{tabular}

表 1 交叉实验结果

Table 1 Results of the cross testing 


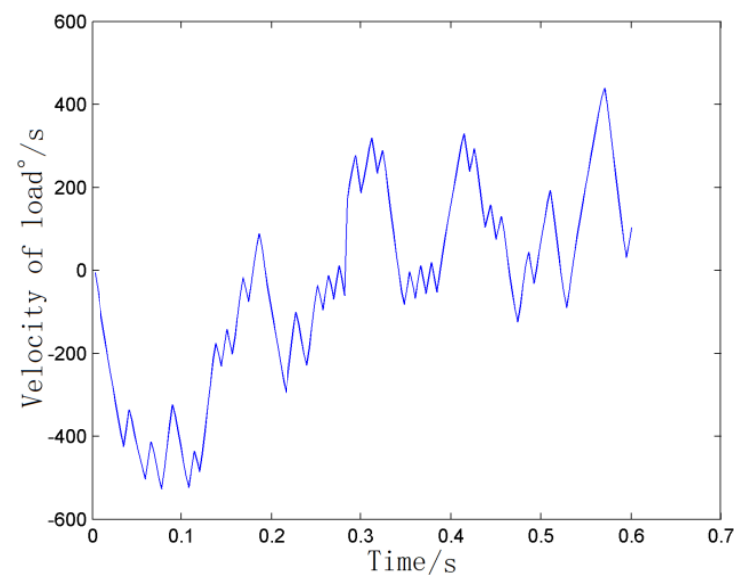

图 7 系统输出速度响应

Fig.7 Velocity output response to system

\section{2 开环验证}

开环验证属于非参数化的验证方法, 检验预测模 型的输入/输出的特性与实际系统输入/输出特性的吻 合程度。在系统带宽和仿真步长的影响下, 系统响应 对不同的信号会呈现各自不同的特点 ${ }^{[8]}$ 。

输入信号是直流信号可以验证两者的时不变性, 稳态值, 动态特性等指标吻合度, 但由于系统有限行 程的限制, 可能会在没有进入稳态前就会停止运动, 所以另外考虑选择不同频率正弦信号进行验证, 在俯 仰方向内来回运动, 频率高的信号变化快, 一个周期 内作用于系统上的电压持续时间低于低频信号作用 时的情况, 所以频率高的信号使对象运动速度的幅值 肯定相对要小。

虽然知道成形滤波器的结构和参数, 但是真实系 统引入的噪声不可测, 仿真时无法直接对模型施加与 真实系统内部白噪声特性一致的白噪声, 也不能盲目 确定其量级和变化情况, 否则会对模型真实输出影响 很大, 在这里只能对比预测模型的理想输出和实际系 统真实输出的误差特征, 控制率保持 $3 \mathrm{~ms}$ 不变, 幅值 都选择 0.1 时, 以阶跃信号作为输入信号激励时, 负 载在俯仰方向上的实时运动位置如图 9, 实际运动速 度输出与预测模型速度输出对比结果如图 10, 预测模 型的动态响应结果如图 11, 当分别以 $5 \mathrm{~Hz}$ 和 $30 \mathrm{~Hz}$ 正弦信号作为输入信号激励时, 实际系统与预测模型 的速度输出对比结果依次如下图的 $12 \sim 13$, 其中表 2 3 依次为两者在不同正弦信号激励下的动态响应 指标对比结果。

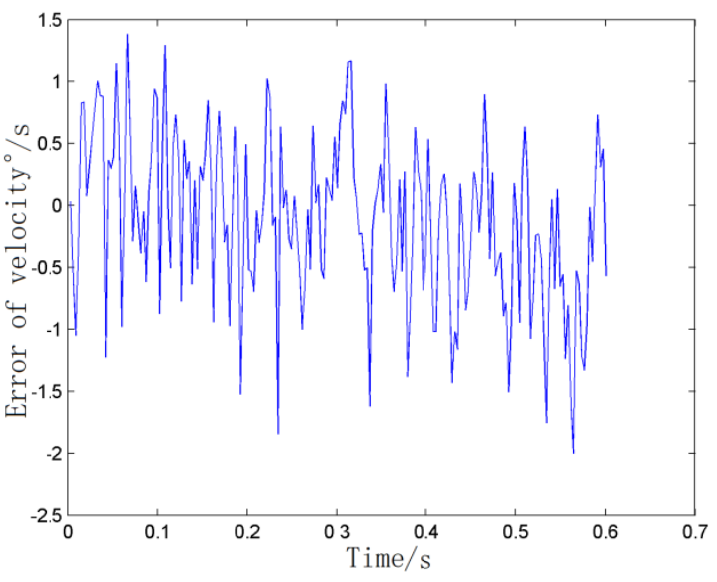

图 8 模型与系统的输出响应误差

Fig.8 Velocity error between model and system

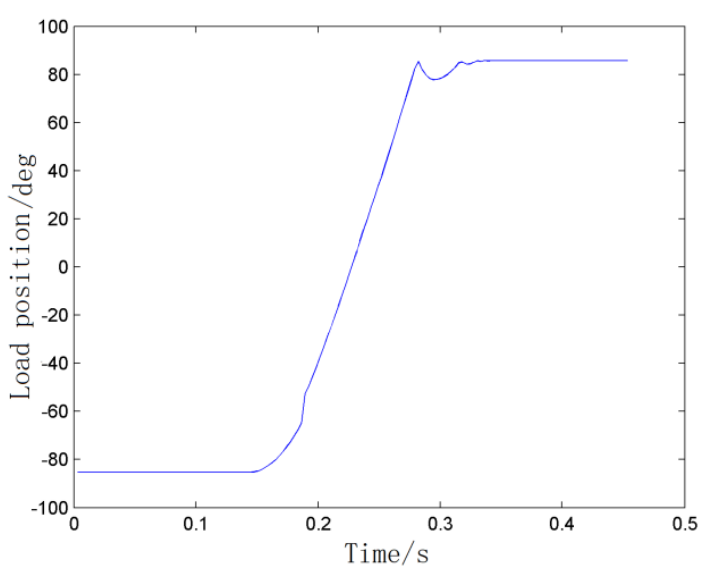

图 9 阶跃信号作用下负载运动位置响应

Fig.9 Load position response when step signal exciting

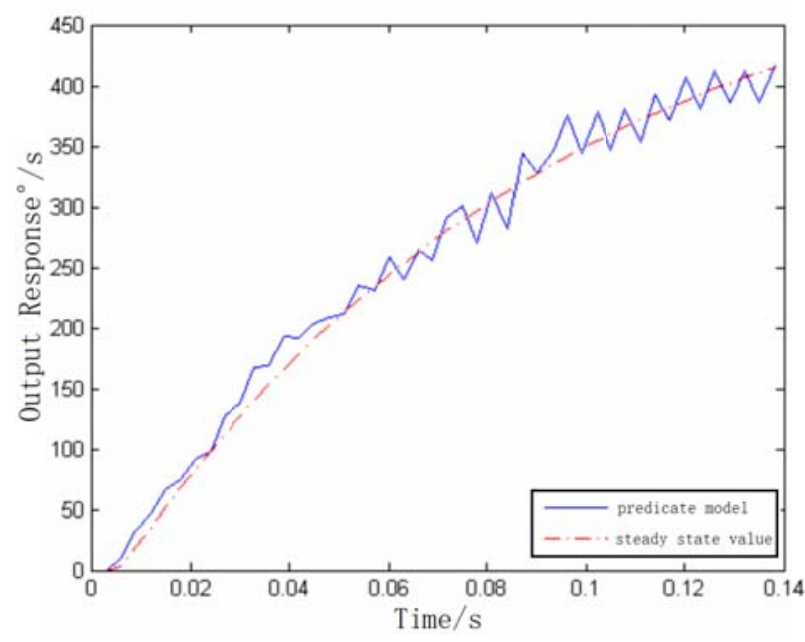

图 10 阶跃信号作用下速度变化

Fig.10 Velocity change response when step signal exciting 


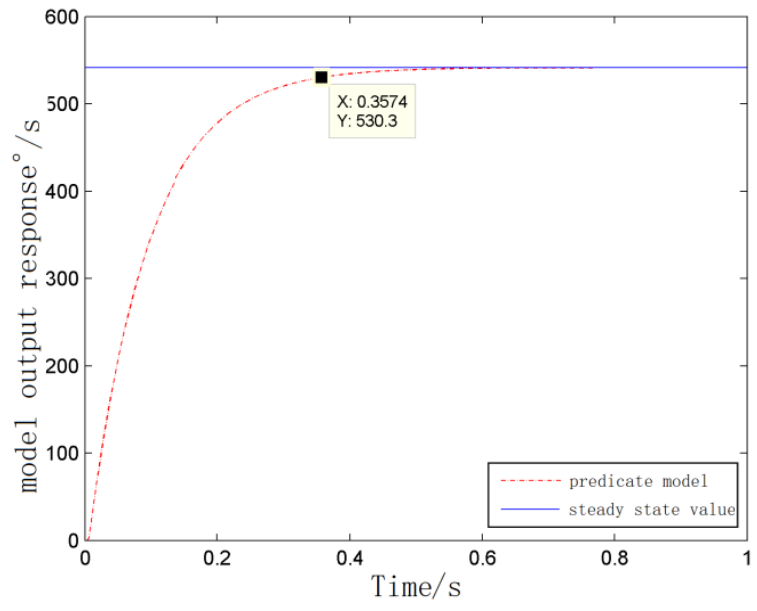

图 11 阶跃信号作用下预测模型动态响应

Fig.11 Dynamic response of predict model when step signal exciting

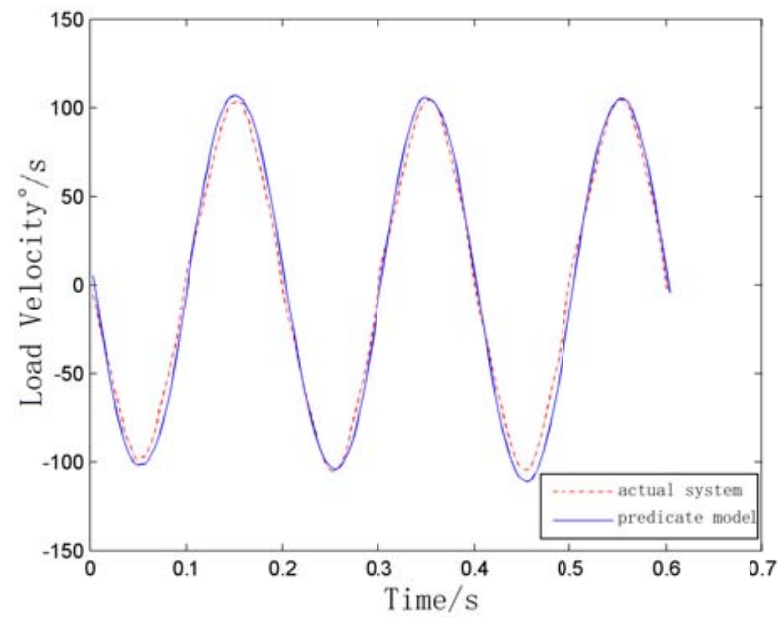

图 $125 \mathrm{~Hz}$ 正弦信号激励下输出响应对比

Fig.12 Output response when $5 \mathrm{~Hz}$ sine signal exciting

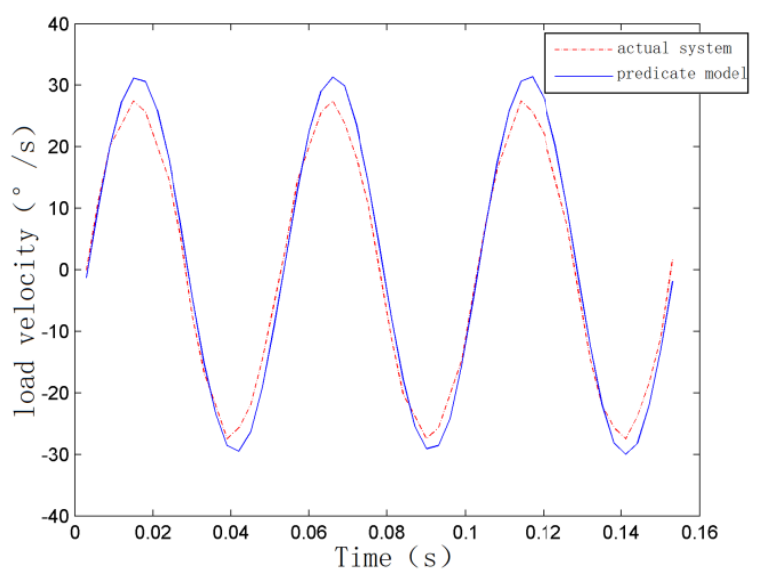

图 $1330 \mathrm{~Hz}$ 正弦信号激励下输出响应对比

Fig.13 Output response when $30 \mathrm{~Hz}$ sine signal exciting
表 $25 \mathrm{~Hz}$ 正弦信号输入时速度响应参数对比表

Table 2 Dynamic parameter of velocity output response when $5 \mathrm{~Hz}$ sine signal exciting

\begin{tabular}{|c|c|c|c|}
\hline & $\begin{array}{l}\text { Actual } \\
\text { system }\end{array}$ & model & error \\
\hline period/s & 0.201 & 0.201 & 0 \\
\hline $\begin{array}{l}\text { Maximum positive velocity } /(\% / s) \\
\text { (three times average) }\end{array}$ & 104.4 & 106. & $1.5 \%$ \\
\hline $\begin{array}{l}\text { Maximum negative velocity } /(\% / s) \\
\text { (three times average) }\end{array}$ & 105.6 & 107.2 & $1.6 \%$ \\
\hline Residual standard deviation & 70.6519 & 71.2618 & 0.6099 \\
\hline
\end{tabular}

Table 3 dynamic parameter of velocity output response when $30 \mathrm{~Hz}$ sine signal exciting

\begin{tabular}{|c|c|c|c|}
\hline & $\begin{array}{l}\text { Actual } \\
\text { system }\end{array}$ & model & error \\
\hline period/s & 0.051 & 0.051 & 0 \\
\hline $\begin{array}{l}\text { Maximum positive velocity } \% \text { s } \\
\text { (three times average) }\end{array}$ & 28.47 & 30.27 & $6.1 \%$ \\
\hline $\begin{array}{l}\text { Maximum negative velocity } \% \text { s } \\
\text { (three times average) }\end{array}$ & 28.03 & 29.45 & $5 \%$ \\
\hline Residual standard deviation & 20.1971 & 21.6987 & 1.5 \\
\hline
\end{tabular}

通过图 9 11 可以看到: 在阶跃信号作用下, 负 载在整个行程范围内, 没有进入稳态过程, 模型的输 出响应要在 $0.36 \mathrm{~s}$ 以后进入稳态区（2\%误差）, 但是 两者在上升过程中的速度曲线贴近, 说明前段运动过 程中, 动态特性基本一致。通过图 12１3 可以看到: 在两种不同频率的正弦信号作用下，模型速度输出响 应与系统速度输出响应的周期和幅值偏差都比较小, 且系统和模型的动态曲线匹配更好。

\section{5 结论}

本文以红外稳定平台伺服系统为研究对象, 先对 其进行了动力学建模, 并通过线性化处理和系统特性 理论分析, 给出了实验方式下参数辨识的具体步骤并 进行了模型有效性的验证。辨识得到的离散化参数模 型通过了参数化的白度测试和非参数化的开环验证, 可见通过增大输入信号增益使负载运行在高速环境 下，并有合理的激励系统带宽范围，确实能够通过实 验辨识得到对实际系统刻画比较精确的参数化模型。

\section{参考文献:}

[1] 予阳. 美国未来侦察骑兵系统[J]. 兵器知识, 2005(10): 43-45. YU Yang. Us future reconnaissance cavalry system[J]. Ordnance 
Knowledge, 2005(10): 43-45.

[2] 陈苗海. 侦察监视车及其光电传感器的发展现状 $[\mathrm{J}]$. 激光与红外, 2005, 33(7): 459-462.

CHEN Miaohai. Development status of reconnaissance and surveillance vehicle and its photoelectric sensor[J]. Laser and Infrared, 2005, 33(7): 459-462.

[3] 朱耘, 韩根甲. 无人机光电探测技术的现状及发展趋势 [J]. 舰船科学 技术, 2004, 26(6): 1-5.

ZHU Yun, HAN Genjia. The current situation and development trend of UAV photoelectric detection technology[J]. Ship Science and Technology, 2004, 26(6):1-5

[4] 廖洪波. 光电稳定平台伺服系统动力学建模与参数辨识[D]. 长沙: 国 防科技大学, 2008 .

LIAO Hongbo. Dynamic modeling and parameter identification of servo system of photoelectric stabilized platform[D]. Changsha: National
Defense University of Science and Technology, 2008.

[5] Debruin J. Control Systems or Mobile Satcom Antennas[J]. IEEE Control Systems Magazine, 2008, 28(1): 87-101.

[6] Guelman M, Kogan A, Kazarian A. Acquisition and Position Control for Inter-satellite Laser Communications[J]. IEEE Trans. Aerospace Electronic Systems, 2004, 40(4): 1239-1249.

[7] Ren Z., Stephens L Laser. Pointing and Tracking using A Completely Electromagnetically Suspended Precision Actuator[J]. AIAA Journal of Guidance, Control and Dynamics, 2006, 29(5): 1235-1239.

[8] 毕永利. 多框架光电平台控制技术研究[D]. 长春：中科院长春光机 所, 2003.

BI Yongli. Research on control technology of multi-frame photoelectric platform[D]. Changchun: Changchun Institute of Optics and mechanics, Chinese Academy of Sciences, 2003. 